\title{
Nanoparticle-Based Membrane Assembly and Silicification in Coacervate Microdroplets as a Route to Complex Colloidosomes
}

\author{
James Fothergill, ${ }^{\dagger}$ Mei Li, ${ }^{\dagger}$ Sean A. Davis, ${ }^{\dagger}$ John A. Cunningham, ${ }^{\dagger}$ and Stephen Mann ${ }^{*}{ }^{\dagger}$ \\ ${ }^{\dagger}$ Centre for Protolife Research, Centre for Organized Matter Chemistry, School of Chemistry, University of Bristol, Bristol BS8 1TS, \\ UK \\ ${ }^{\ddagger}$ School of Earth Sciences, University of Bristol, Life Sciences Building, 24 Tyndall Avenue, Bristol BS8 1TQ , UK
}

Supporting Information

\begin{abstract}
The chemical construction of complex colloidosomes consisting of a molecularly crowded polyelectrolyteenriched interior surrounded by a continuous shell of closely packed silica nanoparticles is studied using optical and fluorescence microscopy, high-resolution X-ray microcomputed tomography, and synchrotron radiation X-ray tomographic microscopy. The colloidosomes are prepared by addition of partially hydrophobic silica nanoparticles to dodecane dispersions of positively or negatively charged coacervate microdroplets consisting of aqueous mixtures of poly(diallyldimethylammonium chloride) (PDDA) and ad-

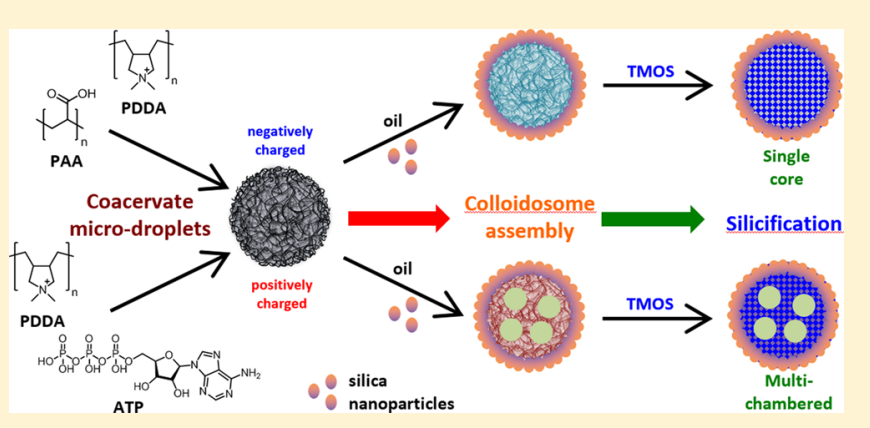
enosine $5^{\prime}$-triphosphate (ATP) or PDDA and poly(acrylic acid) (PAA), respectively. Interfacial assembly of the nanoparticles produces a polydisperse population of well-defined PDDA/PAA droplets with diameters ranging from 50 to $950 \mu \mathrm{m}$. In contrast, reconstruction of the PDDA/ATP coacervate interior occurs on addition of the silica nanoparticles to produce a nanoparticlestabilized oil-in-coacervate-in-oil multiphase emulsion. Transfer of the coacervate-containing colloidosomes into water and replication of their internal structure are achieved by addition of tetramethoxysilane, which serves as both a cross-linking and silicification agent to produce mineralized PDDA/PAA or PDDA/ATP microstructures with a uniform solidified texture or multichambered interior, respectively. The integration of colloidosome and coacervate technologies offers a route to a new type of multifunctional microcompartmentalized system based on the membrane-mediated incarceration of molecularly crowded chemical environments.
\end{abstract}

\section{INTRODUCTION}

The chemical construction of semipermeable, membranebounded microcompartments is increasingly recognized as an important consideration for developing new approaches to integrated materials ensembles, ${ }^{1,2}$ protocell design and construction, ${ }^{3-5}$ and mechanisms related to the origin of life on the early earth. ${ }^{6,7}$ Water-filled, organic membrane-delineated functional microcompartments have been prepared as lipid ${ }^{8-10}$ or polymer vesicles, ${ }^{11-13}$ polyelectrolyte capsules, ${ }^{14,15}$ and hollow protein-polymer based microstructures. ${ }^{16}$ Alternatively, inorganic microcapsules (colloidosomes) have been produced by nanoparticle self-assembly at water/oil interfaces using a range of components. ${ }^{17-19}$ In general, the colloidosome membrane is semipermeable, mechanically robust, and accessible to chemical functionalization. As a consequence, complex multistep processes such as cell-free gene expression, artificial cytoskeletal assembly, enhanced or membrane-gated enzyme-mediated transformations, and protocell growth and division have been investigated in silica nanoparticle based colloidosomes. $^{5,20-22}$

Recent studies have indicated that the spontaneous phase separation of liquid microdroplets in water (coacervation) can serve as an alternative membrane-free system of micro- compartmentalization for protocell modeling. ${ }^{23}$ The coacervate microdroplets are produced by electrostatically mediated complexation of polycationic and polyanionic molecules, such as poly(diallyldimethylammonium chloride) (PDDA) and adenosine 5'-triphosphate (ATP), and comprise chemically enriched, molecularly crowded interiors capable of selective molecular uptake and spatially confined catalysis. While this system has the advantage of simplicity, the absence of an enclosing membrane and low mechanical stability of the coacervate droplets places restrictions on developing the methodology toward technological applications. In response to this challenge, recent investigations have focused on developing water-based routes to membrane-coated coacervate microdroplets involving surface adsorption of colloidal silica particles, ${ }^{24}$ surface complexation of polyoxometallate clusters, ${ }^{25}$ or multilayer molecular assembly of a fatty acid. ${ }^{26}$ In the latter case, self-organization of the fatty acid molecules was achieved without disruption of the coacervate droplets to produce microcompartments with hybrid properties associated with

Received: September 22, 2014

Revised: November 11, 2014

Published: November 12, 2014 
membrane encapsulation of a molecularly complex microenvironment. In contrast, electrostatically mediated assembly of polyanionic polyoxometallate clusters on the surface of PDDA/ ATP coacervate microdroplets produced semipermeable membrane-bounded microcompartments that exhibited a complex three-tiered architecture due to osmotically induced restructuring. ${ }^{25}$

Given these results, and the ongoing challenge to prepare robust microcapsules with increasingly complex architectures such as embedded multicompartments, herein we develop a new approach that involves the stabilization of coacervate microdroplets in oil via nanoparticle-based colloidosome membrane assembly (Figure 1). We demonstrate that a

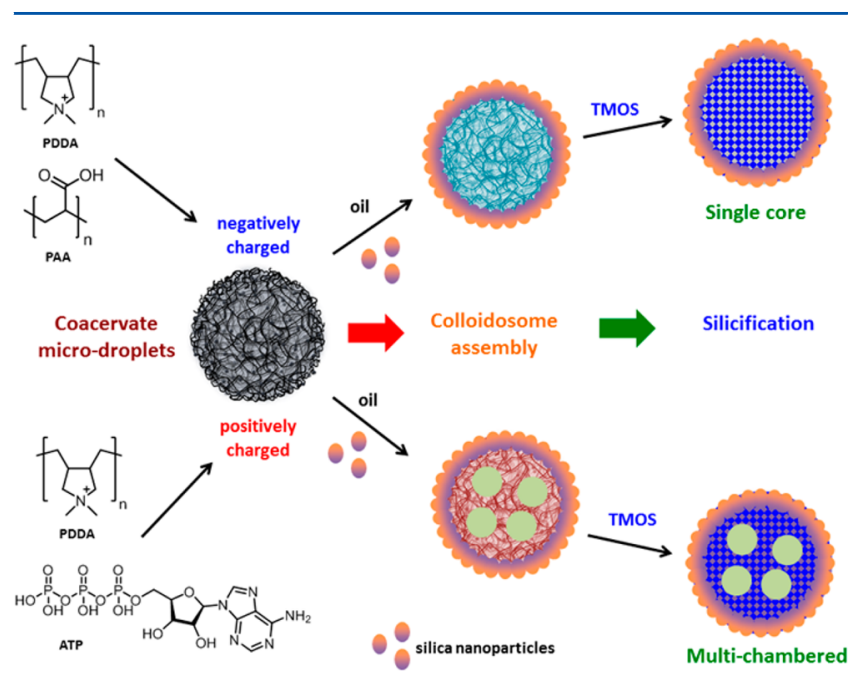

Figure 1. Scheme showing formation of membrane-bounded coacervate droplets and generation of complex colloidosomes.

continuous shell of closely packed partially hydrophobic silica nanoparticles can be assembled around positively (PDDA/ ATP) or negatively charged (PDDA/poly(acrylic acid) (PAA)) coacervate microdroplets dispersed in dodecane. Significantly, assembly of the nanoparticles results in reconstruction of the coacervate interior to give complex colloidosomes with a multicompartmentalized architecture when a net positive charge is associated with the droplets. The membraneencapsulated droplets can be transferred into water by addition of tetramethoxysilane (TMOS), which cross-links the nanoparticle-based shell and permeates the PDDA/PAA or PDDA/ ATP coacervate droplets to produce colloidosomes with a silicified single core or complex multichambered interior, respectively.

\section{EXPERIMENTAL SECTION}

Nanoparticle Assembly and Silicification of Coacervate Microcompartments. Positively charged or negatively charged coacervate microdroplets were prepared at room temperature by mixing aqueous solutions of poly(diallyldimethylammonium chloride) $\left(\right.$ PDDA; $\left.M_{\mathrm{w}}=200 \mathrm{~K}-350 \mathrm{~K}, 1 \mathrm{~mL}, 50 \mathrm{mM}, \mathrm{pH} 8\right)$ and ATP $(250 \mu \mathrm{L}$, $50 \mathrm{mM}, \mathrm{pH} 8)$, or PDDA $(50 \mu \mathrm{L}, 400 \mathrm{mM}, \mathrm{pH} 8))$ and poly(acrylic acid) (PAA, $\left.M_{\mathrm{w}}=5.1 \mathrm{~K}, 800 \mu \mathrm{L}, 100 \mathrm{mM}, \mathrm{pH} 8\right)$, at [PDDA monomer]:[ATP] or [PDDA monomer]:[PAA monomer] molar ratios of $4: 1$ and 1:4, respectively. In both cases, the dispersions were left for $5 \mathrm{~min}$ and then centrifuged $(2300 \mathrm{~g}, 5000 \mathrm{rpm}, 1 \mathrm{~min})$ to separate the coacervate bulk phases from the aqueous supernatants.

Microdroplets of the aqueous coacervates were stabilized in a continuous oil phase by adding small volumes of the bulk coacervate phase $(50 \mu \mathrm{L})$ to $2 \mathrm{~mL}$ of anhydrous dodecane (>99\%), containing a sonicated suspension of partially hydrophobized silica nanoparticles $(10 \mathrm{mg}$, mean diameter $=25 \mathrm{~nm}$, Wacker $\mathrm{GmbH}$, surface functionalities; $\left.50 \% \mathrm{SiOH}, 50 \% \mathrm{Si}\left(\mathrm{CH}_{3}\right)_{2}\right)$. The resulting mixture was vigorously shaken or sonicated in an ultrasonic bath until a turbid water-in-oil Pickering emulsion with an aqueous coacervate:oil volume ratio of 0.025 was produced. The silica nanoparticle-stabilized water droplets were agitated by inversion of the sample vial on a rotary mixer to prevent sedimentation.

Silicification of the nanoparticle-stabilized PDDA/ATP or PDDA/ PAA coacervate microdroplets was undertaken by addition of tetramethoxysilane (TMOS, 98\%, $10 \mu \mathrm{L}, 67.65 \mu \mathrm{mol}$ ) to the prepared Pickering emulsion $(\approx 2 \mathrm{~mL})$. The TMOS:aqueous coacervate volume ratio was $1: 5$, which was commensurate with values used previously to cross-link the silica nanoparticle membrane prior to transfer into water. ${ }^{22}$ The samples were gently agitated for $48-72 \mathrm{~h}$ using a rotary inverter to ensure complete condensation of the organosilane and then left undisturbed in oil or transferred into distilled water using a sequence of acetone/ethanol/water solvents. For this, acetone $(50 \mu \mathrm{L})$ was carefully added to the oil-dispersed suspension, and the mixture left for $30 \mathrm{~min}$ after which another aliquot of acetone $(50 \mu \mathrm{L})$ was added and the vial rotated slowly by hand. The silicified coacervate microdroplets were allowed to sediment, and then the supernatant was decanted and replaced with acetone $(0.5 \mathrm{~mL})$. The suspension was slowly centrifuged at $500 \mathrm{~g}$ for $5 \mathrm{~min}$ and the supernatant decanted. This was repeated with a 50:50 ethanol:water mixture and then finally with deionized water. The mineralized droplets readily sedimented from the aqueous dispersions. No changes in microstructure were observed for samples stored for at least 6 months.

Characterization. Bright field, phase contrast, and fluorescence images of silica-treated PDDA/ATP or PDDA/PAA coacervate droplets dispersed in oil or water were recorded between coverslips in a capillary cell or Petri dish using a Leica DMI 3000B fluorescence optical microscope. Droplets were stained with fluorescent dyes, including $2^{\prime}, 3^{\prime}$-O-trinitrophenyladenosine-5' $5^{\prime}$-triphosphate triethylammonium salt (TNP-ATP, Jena Bioscience) and 9-diethylamino-5benzo $[\alpha]$ phenoxazinone (Nile red, Sigma-Aldrich), and fluorescein isothiocyanate (FITC)-labeled silica nanoparticles. The fluorescent nanoparticles were prepared by adding (3-aminopropyl)triethoxysilane $\left(10 \mu \mathrm{L}, 10 \mathrm{mg} \mathrm{mL}^{-1}\right.$ in ethanol) and triethylamine $\left(10 \mu \mathrm{L}, 5 \mathrm{mg} \mathrm{mL}^{-1}\right.$ in ethanol) to a dispersion of silica nanoparticles $(25 \mathrm{mg}$ in $2 \mathrm{~mL}$ of ethanol) and then stirring the suspension for $24 \mathrm{~h}$ at room temperature, followed by dropwise addition of fluorescein isothiocyanate $\left(20 \mu \mathrm{L}, 1 \mathrm{mg} \mathrm{mL}^{-1}\right.$ in ethanol). The suspension was then stirred for another $5 \mathrm{~h}$ before purification by centrifugation and washing in ethanol. Fluorescence images were obtained by laser excitation with the following filter settings and excitation wavelengths: I3 (blue, 450$490 \mathrm{~nm}$ ), N2.1 (green, 515-560 nm), D (violet, 355-425 nm), A $(\mathrm{UV}, 340-380 \mathrm{~nm})$. Images were obtained in a Leica Application Suite and edited and converted to jpeg files in ImageJ or FIJI.

Samples of silica-treated coacervate droplets in oil or water were mounted onto a glass slide and imaged by confocal fluorescence microscopy (Leica AOBS SP2 confocal imaging system). Confocal slices were obtained from TNP-ATP-doped samples in fluorescence and bright field mode using a laser excitation of $488 \mathrm{~nm}$. TNP-ATP has a broad emission and was imaged as blue or green fluorescence. Confocal wavelength scans were also performed by measuring the gray-value intensity of pixels in the whole image or in restricted regions of interest. Fluorescein-labeled silica nanoparticle-stabilized microstructures were imaged at $496 \mathrm{~nm}$.

Fluorescence profiles were obtained using a Jasco FP-6500 spectrofluorometer. Samples were loaded into reduced volume quartz cuvettes, and the excitation profile was initially obtained. The spectra were then optimized by small variations in the excitation wavelength to achieve maximum emission intensity.

Zeta-potential and dynamic light scattering (DLS) studies were undertaken on diluted aqueous suspensions using a Malvern Zetasizer Nano instrument. DLS measurements were obtained at a backscatter angle of $173^{\circ}$, with refractive indices of 1.544 (silica), 1.330 (water), and 1.361 (ethanol) at $25^{\circ} \mathrm{C}$. Average values were obtained from three measurements. Surface topography information was obtained using a 
Bruker Multimode AFM with a Veeco Nanoscope V controller and picoforce extender. The samples were dried in air, mounted onto freshly cleaved mica, and imaged in air in tapping mode. Some samples for SEM were embedded in epoxy resin (araldite CY 212 (M), dodecenylsuccinic anhydride (DDSA) and N,N-benzydimethylamine (BDMA, 3\%; Agar Scientific). Thermogravimetric analysis (TGA) was undertaken from room temperature to $1100{ }^{\circ} \mathrm{C}$ using a Netzsch simultaneous thermal analysis STA409EP instrument. Samples were loaded into an aluminum oxide crucible, and the initial mass was recorded. First derivatives were obtained to determine peak temperatures.

Three-dimensional analyses of the internal microarchitecture of the silicified microstructures were obtained by high-resolution X-ray microcomputed tomography $(\mu-\mathrm{CT})$ and synchrotron radiation $\mathrm{X}$ ray tomographic microscopy (SRXTM). Samples for $\mu$-CT were wrapped in low-density polyethylene (household plastic wrap) and mounted into a SkyScan 1172 (Kontich, Belgium) capable of imaging with a voxel size of $4.8 \mu \mathrm{m}^{3}$. Scans were obtained on dry and wet samples, although differences in material density were more pronounced for predried samples. The images were reconstructed using SkyScan software and visualized in ImageJ.

Specimens for SRXTM were dried down in air and mounted on 3 $\mathrm{mm}$ brass stubs using clear nail varnish. The samples were then characterized on the TOMCAT beamline at the Swiss Light Source, Paul Scherrer Institute, Villigen, Switzerland. Measurements were obtained using $\times 10$ and $\times 20$ objective lenses at $10-15 \mathrm{keV}$, with voxel resolutions of 0.74 and $0.36 \mu \mathrm{m}$, respectively. Each data set was reconstructed from projections at $0.12^{\circ}$ intervals between $0^{\circ}$ and $180^{\circ}$. The reconstructed images were then prepared using VSG Avizo 8, in which the silica phase was manually labeled. The labels were then converted into surface plots and false colored to enhance visualization.

\section{RESULTS AND DISCUSSION}

Positively or negatively charged coacervate microdroplets were prepared at room temperature by mixing aqueous solutions of PDDA $\left(M_{\mathrm{w}}=200 \mathrm{~K}-350 \mathrm{~K}, 1 \mathrm{~mL}, 50 \mathrm{mM}, \mathrm{pH} 8\right)$ and ATP (250 $\mu \mathrm{L}, 50 \mathrm{mM}, \mathrm{pH} 8)$, or PDDA $(50 \mu \mathrm{L}, 400 \mathrm{mM}, \mathrm{pH} 8))$ and PAA $\left(M_{\mathrm{w}}=5.1 \mathrm{~K}, 800 \mu \mathrm{L}, 100 \mathrm{mM}, \mathrm{pH} 8\right)$, at [PDDA monomer]:[ATP] or [PDDA monomer]:[PAA monomer] molar ratios of $4: 1$ and $1: 4$, respectively. Zeta-potential measurements gave values of +31 or $-28 \mathrm{mV}$ for aqueous suspensions of the PDDA/ATP or PDDA/PAA microdroplets, respectively, at $\mathrm{pH} 8$ (Figure $\mathrm{S} 1$ ). The spontaneous assembly of an ultrathin nanoparticle-based membrane on the surface of coacervate microdroplets was undertaken by dispersing small volumes $(50 \mu \mathrm{L})$ of the centrifuged aqueous bulk coacervate phases in $2 \mathrm{~mL}$ of anhydrous dodecane containing a sonicated suspension of partially hydrophobized silica nanoparticles (10 $\mathrm{mg}$, mean diameter $=25 \mathrm{~nm})$. The nanoparticles comprised approximately $50 \% \mathrm{SiOH}$ and $50 \% \mathrm{Si}\left(\mathrm{CH}_{3}\right)_{2}$ surface functional groups and exhibited a zeta-potential of $-4 \mathrm{mV}$ when dispersed in water at $\mathrm{pH} 7.5$ (Figure S1). Vigorous shaking or sonication in an ultrasonic bath of the coacervate/oil mixture with a volume ratio of 0.025 produced a turbid water-in-oil Pickering emulsion comprising nanoparticle-stabilized coacervate droplets that when left unstirred for 5 min sedimented to form a layer of discrete spherical microstructures (colloidosomes) (Figure S2).

Optical microscopy images recorded in oil of the colloidosomes prepared from the negatively charged PDDA/ PAA coacervate showed a polydisperse population of welldefined droplets with diameters ranging from 50 to $950 \mu \mathrm{m}$ (mean $=380 \pm 10 \mu \mathrm{m})$ and corresponding to a mean encapsulated volume of $30 \mathrm{~nL}$ (Figure 2A). Water-soluble dye molecules sequestered into the coacervate phase prior to nanoparticle assembly were readily imaged by fluorescence microscopy, indicating that the guest molecules were retained

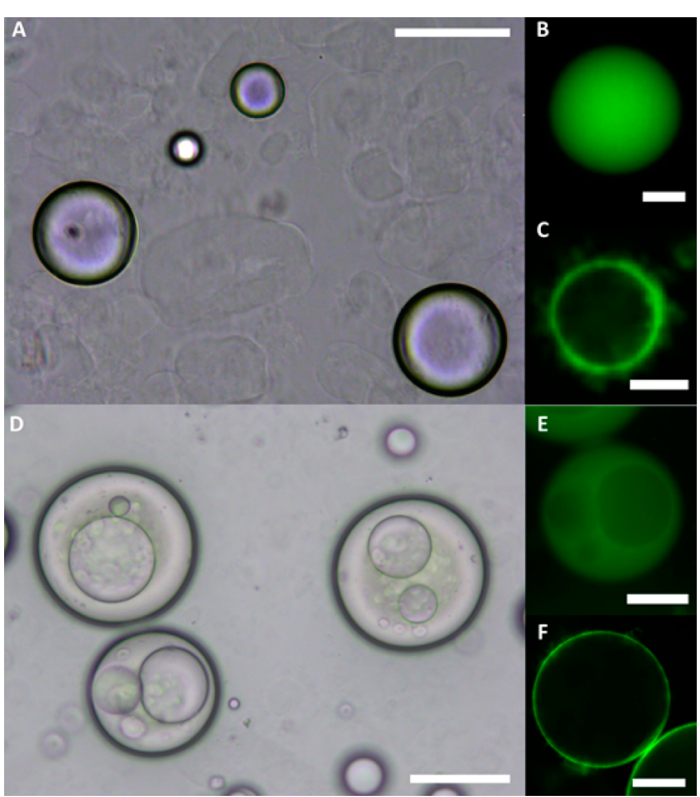

Figure 2. (A-C) Silica nanoparticle stabilization of negatively charged aqueous PDDA/PAA coacervate microdroplets in oil. (A) Optical microscopy image showing homogeneous single coacervate droplets after nanoparticle surface assembly, scale bar $=100 \mu \mathrm{m}$, (B) fluorescence microscopy image showing retention of water-soluble green fluorescent dye (calcein) within the droplet interior, scale bar = $25 \mu \mathrm{m}$, and (C) FITC-labeled silica nanoparticle-stabilized droplet showing green fluorescent ring corresponding to a continuous inorganic membrane surrounding the coacervate microphase, scale bar $=25 \mu \mathrm{m}$. (D-F) Silica nanoparticle stabilization of positively charged aqueous PDDA/ATP coacervate microdroplets in oil. (D) Optical microscopy image showing nanoparticle-stabilized coacervate droplets with internal substructures, scale bar $=200 \mu \mathrm{m}$, (E) fluorescence microscopy image showing spatial localization of aqueous calcein within a single coacervate droplet, confirming that the encapsulated substructures are oil microdroplets; scale bar $=100$ $\mu \mathrm{m}$, and (F) fluorescence microscopy image showing the presence of a continuous inorganic membrane surrounding a single FITC-labeled silica nanoparticle-stabilized droplet, scale bar $=100 \mu \mathrm{m}$.

within the Pickering emulsions (Figure 2B). The fluorescence images showed a uniform distribution of dye molecules throughout the coacervate interior of the stabilized droplets. Significantly, by using fluorescein isothiocyanate (FITC)labeled silica nanoparticles, we confirmed that the coacervate droplets were enclosed in a continuous ultrathin inorganic membrane (Figure 2C). Similar experiments using the positively charged PDDA/ATP coacervate also produced a polydisperse Pickering emulsion of silica nanoparticle-stabilized aqueous polymer-nucleotide droplets in oil. Although the positively charged droplets were similar in shape and diameter (range $50-450 \mu \mathrm{m}$; mean $=240 \pm 5 \mu \mathrm{m}$ ) to those produced using the negatively charged PDDA/PAA coacervate, the former were structurally complex. Optical microscopy images showed individual droplets with multiple internalized compartments that remained stable and did not coalesce within the encapsulated coacervate phase (Figure 2D). The number of entrapped droplets ranged from a few relatively large structures around $100 \mu \mathrm{m}$ or so in diameter to arrays of discrete droplets often only a few tens of micrometers in size (Figure S3). Solubilization of a water-soluble fluorescent dye (calcein) in the coacervate phase confirmed that the internalized droplets were dodecane and that an oil-in-coacervate-in-oil multiphase 
emulsion was produced under these conditions (Figure 2E). Fluorescence microscopy images of samples produced using fluorescently labeled silica nanoparticles showed a bright outer membrane consistent with the colloidsome microarchitecture (Figure 2F). The images did not however reveal the presence of nanoparticles at the internalized oil/water interface. We attributed the apparent absence of an inorganic membrane around each of the internalized oil droplets to a highly reduced fluorescence intensity in the interior of the coacervate phase. However, we could not rule out the possibility that the internalized oil microdroplets were stabilized by interfacial adsorption of PDDA or were physically immobilized in the interior due to the viscosity of the coacervate medium.

The above results indicated that both positively and negatively charged aqueous coacervates can be stabilized as discrete droplets in dodecane by the spontaneous interfacial assembly of a continuous membrane comprising at least a monolayer of closely packed silica nanoparticles. Assembly of the surface-active nanoparticles on the negatively charged PDDA/PAA microphase produced an inorganic membrane sufficiently stable to withstand the transient ingress of oil microdroplets during formation of the new interface. In contrast, the nanoparticle-stabilized interface generated at the boundary of the positively charged PDDA/ATP phase was less resilient to the influx of oil, suggesting that electrostatic interactions between the cationic polymer and partially negatively charged silica nanoparticles were responsible for destabilization of the colloidosome membrane under these conditions.

Given the above observations, we undertook experiments to determine whether the silica nanoparticle-stabilized coacervate microdroplets could be transferred from oil into water with retention of the outer membrane shell, and in the case of the PDDA/ATP system without loss of the multichambered microstructure. For this, we added small amounts of TMOS to the Pickering emulsion to induce hydrolysis and condensation reactions specifically at the water/oil interface such that the silica nanoparticles became cross-linked into a structurally coherent membrane that remained intact during transfer into water. Specifically, we used a TMOS:aqueous coacervate volume ratio of 1:5 because previous studies indicated that under these conditions cross-linking occurred without silica penetration into the aqueous-filled interior. ${ }^{22}$ Surprisingly, although the silica-coated PDDA/PAA coacervate droplets could be successfully cross-linked and transferred into water without loss of membrane structure (Figure 3A), optical microscopy images (Figure 3B), SEM backscattered images from ultramicrotomed sectioned samples (Figure S4), and synchrotron radiation $\mathrm{X}$-ray tomographic microscopy (SRXTM) 3-D reconstructions (Figure 3C) indicated that addition of TMOS resulted in complete solidification of the encapsulated aqueous polymer mixture. The solidified cores were relatively uniform in texture and nonporous at the micrometre scale. Arrays of voids were often observed in the near-surface regions of the silicified collodosomes (Figure 3C). Analysis of the colloidosomes by powder X-ray diffraction and ATR-FTIR spectroscopy confirmed the presence of amorphous silica, PDDA, and PAA in the mineralized microstructures (Figures S5 and S6). We attributed the formation of the solid silicified interior to facile penetration of the organosilane into the PDDA/PAA coacervate matrix, suggesting that this process was competitive with TMOS hydrolysis/condensation at the oil/water interface. One possibility is that the reactivity of

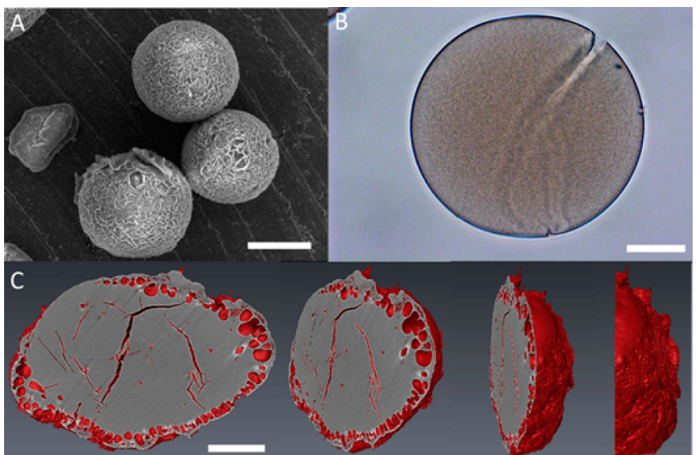

Figure 3. Silicification and transfer into water of colloidosomes with uniform aqueous PDDA/PAA coacervate interiors. (A) SEM image of dried colloidosomes after reaction with TMOS in oil and phase transfer showing intact microspheres with highly textured surface; scale bar $=100 \mu \mathrm{m}$. (B) Optical microscope image showing single silicified colloidosome with mineralized interior; scale bar $=50 \mu \mathrm{m}$. (C) SRXTM 3-D reconstruction of a single silicified PDDA/PAA colloidosome showing set of four rotated video images revealing homogeneous solidification of the interior, arrays of subsurface voids, and roughened outer membrane; scale bar $=200 \mu \mathrm{m}$.

TMOS is inhibited at the surface of the silica nanoparticles due to polyelectrolyte adsorption, and as a consequence unreacted TMOS molecules diffuse from the water/oil interface into the aqueous coacervate interior where they slowly react to produce a silica/polymer matrix enclosed in a shell of silica nanoparticles.

Similar experiments involving the addition of TMOS and subsequent transfer into water were undertaken with the silica nanoparticle-coated PDDA/ATP colloidosomes. SEM images of the dried transferred samples showed mineralized microspheres with a semispherical morphology (Figure 4A) and highly roughened outer surface that consisted of closely packed clusters of silica nanoparticles (Figure 4B). FTIR spectra confirmed the presence of silica, PDDA, and ATP in association with the colloidosomes (Figure S7), and TGA profiles gave a silica content of ca. 70 wt \% (Figure S8). Colloidosomes prepared using FITC-labeled silica nanoparticles indicated that the silica nanoparticle membrane remained intact after addition of TMOS and phase transfer (Figure 4C,D). To determine the spatial distribution of components in the multichambered microarchitectures, we doped the PDDA/ATP coacervate with $0.2 \mathrm{~mol} \%$ of the fluorescent mononuclotide $2^{\prime}, 3^{\prime}-\mathrm{O}$ trinitrophenyladenosine- $5^{\prime}$-triphosphate triethylammonium salt (TNP-ATP) prior to TMOS addition. The fluorescence images clearly showed that the encapsulated PDDA/ATP coacervate phase remained subdivided by oil microdroplets after addition of TMOS to the oil phase (Figure 4E,F) as well as after transfer of the samples into water (Figure 4G,H). Significantly, backscattered SEM images and corresponding EDX analyses of colloidosomes embedded and sectioned in resin showed a close spatial correlation between the $\mathrm{Si}$ and $\mathrm{P}$ maps in the continuous regions of the aqueous coacervate phase, confirming that the polymer-nucleotide matrix was silicified after addition of TMOS (Figure 4I-K). This was consistent with 3-D reconstructions of the microstructures obtained from $\mu$-CT (Figure 4L) and SRXTM imaging (Figure $4 \mathrm{M})$, which showed mineralized replicas with an interconnected structural framework and high level of discrete void spaces (Figure $4 \mathrm{~N}$ ). The multichambered interior comprised a random arrangement of large and small voids that were quasi- 


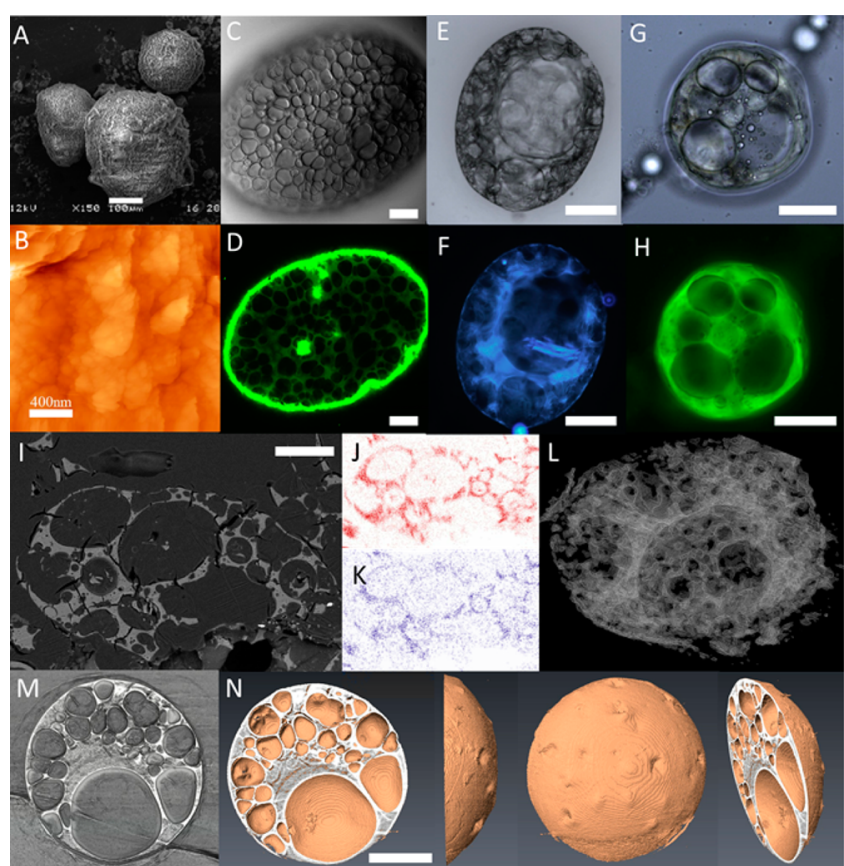

Figure 4. Silicification and phase transfer of colloidosomes prepared from aqueous PDDA/ATP coacervate microdroplets. (A) SEM image of dried colloidosomes after reaction with TMOS in oil and phase transfer showing intact microspheres with highly textured surface; scale bar $=100 \mu \mathrm{m}$. (B) Tapping mode AFM image showing roughened, cross-linked nanoparticle membrane; scale bar $=400 \mu \mathrm{m}$. (C, D) Confocal bright field (C) and corresponding fluorescence (D) microscopy images of a silicified and transferred colloidosome stabilized with FITC-labeled silica nanoparticles showing retention of the outer membrane (intense green fluorescence); scale bar $=100$ $\mu \mathrm{m}$. (E-H) Optical (E, G) and fluorescence (F, H) microscopy images of individual colloidosomes after addition of TMOS showing retention of the multichambered interior for silicified samples dispersed in oil (E, F) or water $(\mathrm{G}, \mathrm{H})$. Fluorescence in (F) (blue) and $(\mathrm{G})$ (green) originates from water-soluble TNP-ATP, which is present in the encapsulated coacervate phase but not within the entrapped oil microdroplets; scale bars $200 \mu \mathrm{m}$ (E, F) and $100 \mu \mathrm{m}(\mathrm{G}$, $\mathrm{H})$. (I-K) Resin-embedded, sectioned sample showing a single colloidosome with complex internal microstructure; composite backscattered SEM (scale bar $=100 \mu \mathrm{m})(\mathrm{I})$ and corresponding EDX analysis maps for $\mathrm{Si}(1.7 \mathrm{keV})(\mathrm{J})$ and $\mathrm{P}(2 \mathrm{keV})(\mathrm{K})$ indicate that ATP and silica are colocated within the encapsulated coacervate matrix. (L, M) 3-D reconstructions of single silicified colloidosomes using $\mu$-CT (L) and SRXTM (M) imaging. (N) Set of four rotated SRXTM video images showing details of multichambered interior and textured outer membrane surface; scale bar $=100 \mu \mathrm{m}$.

spherical in shape and which did not penetrate the outer silica nanoparticle membrane.

\section{CONCLUSIONS}

In conclusion, complex colloidosomes consisting of a molecularly crowded polyelectrolyte-enriched interior surrounded by a continuous shell of silica nanoparticles have been prepared by interfacial stabilization of coacervate microdroplets suspended in dodecane. Self-assembly of an outer membrane of silica nanoparticles occurs spontaneously at the surface of positively charged (PDDA/ATP) or negatively charged (PDDA/PAA) droplets. In the former case, small oil droplets are also internalized in the coacervate phase to produce colloidosomes with a multicompartmentalized interior. Transfer of the colloidosomes into water and replication of the internal structure are achieved by addition of TMOS, which serves as both a cross-linking and silicification agent to produce mineralized microstructures with composite structure and form.

Integration of colloidosome and coacervate technologies offers a route to a new type of multifunctional microcompartmentalized system that is based on the membranemediated incarceration of molecularly crowded chemical environments. In contrast with alternative approaches to membrane-coated coacervate droplets based on the surface binding of polyoxometallate clusters ${ }^{25}$ or multilayer molecular assembly of a fatty acid, ${ }^{26}$ use of silica nanoparticles as a surface-active building block provides an opportunity to construct colloidosomes with interiors that are structurally complex, multichambered, and chemically enriched. As such, the complex colloidosomes described herein could have potential applications in diverse areas of colloid science, biomimetic materials research, and microreactor technology.

\section{ASSOCIATED CONTENT}

\section{S Supporting Information}

Experimental procedures and additional figures. This material is available free of charge via the Internet at http://pubs.acs.org.

\section{AUTHOR INFORMATION}

\section{Corresponding Author}

*E-mail: s.mann@bristol.ac.uk (S.M.).

\section{Notes}

The authors declare no competing financial interest.

\section{ACKNOWLEDGMENTS}

We thank the ERC Advanced Grant scheme for financial support and the University of Bristol for the award of a $\mathrm{PhD}$ studentship to J.F. We acknowledge the Chemistry Imaging Facility for SEM and AFM studies, the Wolfson Bioimaging Facility (Dr. A. Leard) for assistance with confocal microscopy, Prof. P. Donoghue for providing beamline time, and Prof. J. Price and P. Delisser for their support in running $\mu$-CT analysis.

\section{REFERENCES}

(1) Städler, B.; Price, A. D.; Chandrawati, R.; Hosta-Rigau, L.; Zelikin, A. N.; Caruso, F. Polymer Hydrogel Capsules: En Route toward Synthetic Cellular Systems. Nanoscale 2009, 1, 68-73.

(2) Renggli, K.; Baumann, P.; Langowska, K.; Onaca, O.; Bruns, N.; Meier, W. Selective and Responsive Nanoreactors. Adv. Funct. Mater. 2011, 21, 1241-1259.

(3) Luisi, P. L.; Ferri, F.; Stano, P. Approaches to Semi-Synthetic Minimal Cells: A Review. Naturwissenschaften 2006, 93, 1-13.

(4) Noireaux, V.; Maeda, Y. T.; Libchaber, A. Development of an Artificial Cell, from Self-Organization to Computation and SelfReproduction. Proc. Natl. Acad. Sci. U. S. A. 2011, 108, 3473-3480.

(5) Li, M.; Huang, X.; Tang, T.-Y. D.; Mann, S. Synthetic Cellularity Based on Non-Lipid Micro-Compartments and Protocell Models. Curr. Opin. Chem. Biol. 2014, 22, 1-11.

(6) Monnard, P.-A.; Deamer, D. W. Membrane Self-Assembly Processes: Steps toward the First Cellular Life. Anat. Rec. 2002, 268, 196-207.

(7) Rasmussen, S.; Chen, L. H.; Nilsson, M.; Abe, S. Bridging Nonliving and Living Matter. Artif. Life 2003, 9, 269-316.

(8) Mansy, S. S.; Schrum, J. P.; Krishnamurthy, M.; Tobé, S.; Treco, D. a; Szostak, J. W. Template-Directed Synthesis of a Genetic Polymer in a Model Protocell. Nature 2008, 454, 122-125.

(9) Martini, L.; Mansy, S. S. Cell-like Systems with Riboswitch Controlled Gene Expression. Chem. Commun. 2011, 47, 1073410736. 
(10) Nourian, Z.; Danelon, C. Linking Genotype and Phenotype in Protein Synthesizing Liposomes with External Supply of Resources. ACS Synth. Biol. 2013, 2, 186-193.

(11) Peters, R. J. R. W.; Marguet, M.; Marais, S.; Fraaije, M. W.; van Hest, J. C. M.; Lecommandoux, S. Cascade Reactions in Multicompartmentalized Polymersomes. Angew. Chem., Int. Ed. 2014, 53, $146-150$.

(12) van Dongen, S. F. M.; de Hoog, H.-P. M.; Peters, R. J. R. W.; Nallani, M.; Nolte, R. J. M.; van Hest, J. C. M. Biohybrid Polymer Capsules. Chem. Rev. 2009, 109, 6212-6274.

(13) Peters, R. J. R. W.; Louzao, I.; van Hest, J. C. M. From Polymeric Nanoreactors to Artificial Organelles. Chem. Sci. 2012, 3, 335-342.

(14) Retention, C.; Chandrawati, R.; Hosta-rigau, L.; Vanderstraaten, D.; Lokuliyana, S. A.; Sta, B.; Albericio, F.; Caruso, F. Engineering Advanced Capsosomes: Maximizing the Number of TemperatureTriggered Reaction. ACS Nano 2010, 4, 1351-1361.

(15) Chandrawati, R.; Caruso, F. Biomimetic Liposome- and Polymersome-Based Multicompartmentalized Assemblies. Langmuir 2012, 28, 13798-13807.

(16) Huang, X.; Li, M.; Green, D. C.; Williams, D. S.; Patil, A. J.; Mann, S. Interfacial Assembly of Protein-Polymer Nano-Conjugates into Stimulus-Responsive Biomimetic Protocells. Nat. Commun. 2013, 4, 2239.

(17) Subramaniam, A. B.; Wan, J.; Gopinath, A.; Stone, H. A. SemiPermeable Vesicles Composed of Natural Clay. Soft Matter 2011, 7, $2600-2612$.

(18) Bachinger, A.; Kickelbick, G. Pickering Emulsions Stabilized by Anatase Nanoparticles. Monatsh. Chem. 2010, 141, 685-690.

(19) Zgheib, N.; Putaux, J.; Thill, A.; D’Agosto, F.; Lansalot, M.; Bourgeat-Lami, E. Stabilization of Miniemulsion Droplets by Cerium Oxide Nanoparticles: A Step toward the Elaboration of Armored Composite Latexes. Langmuir 2012, 28, 6163-6174.

(20) Wang, Z.; van Oers, M. C. M.; Rutjes, F. P. J. T.; van Hest, J. C. M. Polymersome Colloidosomes for Enzyme Catalysis in a Biphasic System. Angew. Chem., Int. Ed. 2012, 51, 10746-10750.

(21) Wu, C.; Bai, S.; Ansorge-Schumacher, M. B.; Wang, D. Nanoparticle Cages for Enzyme Catalysis in Organic Media. Adv. Mater. 2011, 23, 5694-5699.

(22) Li, M.; Harbron, R.; Weaver, J.; Binks, B.; Mann, S. Electrostatically Gated Membrane Permeability in Inorganic Protocells. Nat. Chem. 2013, 5, 529-536.

(23) Koga, S.; Williams, D. S.; Perriman, A. W.; Mann, S. PeptideNucleotide Microdroplets as a Step towards a Membrane-Free Protocell Model. Nat. Chem. 2011, 3, 720-724.

(24) McKenna, B. J.; Birkedal, H.; Bartl, M. H.; Deming, T. J.; Stucky, G. D. Micrometer-Sized Spherical Assemblies of Polypeptides and Small Molecules by Acid-Base Chemistry. Angew. Chem., Int. Ed. 2004, 43, 5652-5655.

(25) Williams, D. S.; Patil, A. J.; Mann, S. Spontaneous Structuration in Coacervate-Based Protocells by Polyoxometalate-Mediated Membrane Assembly. Small 2014, 10, 1830-1840.

(26) Tang, T.-Y. D.; Che Hak, C. R.; Thompson, A. J.; Kuimova, M. K.; Williams, D. S.; Perriman, A. W.; Mann, S. Fatty Acid Membrane Assembly on Coacervate Microdroplets as a Step towards a Hybrid Protocell Model. Nat. Chem. 2014, 6, 527-533. 